\section{Análises cariotípicas em Leporellus vittatus e Leporinus striatus (Teleostei, Characiformes, Anostomidae) da Bacia do Alto Paraguai, Mato Grosso, Brasil}

\author{
Karyotypic analysis in Leporellus vittatus and Leporinus \\ striatus (Teleostei, Characiformes, Anostomidae) from \\ Upper Paraguay Basin, Mato Grosso State, Brazil
}

Diones Krinski $^{[a]}$, Carlos Suetoshi Miyazawa ${ }^{[b]}$

\begin{abstract}
Resumo
Os peixes da família Anostomidae (Characiformes) representam um dos grupos mais importantes da ictiofauna de água doce da América do Sul, com muitas espécies de alto valor econômico em termos de pesca, piscicultura e também como espécies ornamentais. Esta família possui 12 gêneros com 138 espécies descritas e cerca de 25 espécies novas ainda não descritas. Quando consideradas as pesquisas citogenéticas realizadas com esta família, a maioria dos estudos foram realizados com grupos de peixes com pintas, sendo poucas as análises com os grupos de listras. Cromossomicamente, os anostomídeos com pintas representa um dos grupos de peixes mais bem estudados com espécies apresentando cromossomos sexuais do tipo ZZ/ ZW e cromossomos supranumerários, embora a maioria não apresente cromossomos sexuais. Deste modo, este trabalho teve como objetivo auxiliar no conhecimento citogenético deste grupo de peixes através de análise cromossômica das espécies Leporellus vittatus e Leporinus striatus. Ambas as espécies analisadas mostraram valor diploide modal de $2 n=54$ cromossomos; metacêntricos e submetacêntricos e número fundamental igual a 108. A heterocromatina constitutiva esteve presente em pequenos blocos intersticiais ou proximais aos telômeros da maioria dos cromossomos de $L$. striatus, havendo um bloco heterocromático bem marcado na região pericentromérica de um par de submetacêntrico, corroborando com a região organizadora de nucléolos (Ag-RON) do tipo simples. Em L. vittatus a heterocromatina constitutiva esteve presente em blocos heterocromáticos bem marcados nos centrômeros de alguns cromossomos, enquanto outros apresentaram marcações conspícuas na região pericentromérica e a Ag-RON mostrou-se evidente em um par cromossômico na região telomérica dos braços longos de um cromossomo submetacêntrico. Os resultados obtidos corroboram com outros trabalhos, mostrando que este grupo possui de fato um cariótipo estável, o que é esperado em peixes que apresentam alta vagilidade.
\end{abstract}

Palavras-chave: Anostomídeos. Citogenética. Cromossomos. Ictiofauna Neotropical. Região Organizadora de Nucléolos (Ag-RONs).

\begin{abstract}
Fishes of the family Anostomidae (Characiformes) represent one of the most important groups of freshwater fishes of South America, with many species of high economic value in terms of fishing, fish farms and as ornamental species. This family has 12 genera with 138 described species and about 25 new species not yet described. When considered the cytogenetic researches conducted with this family the majority of studies was performed with groups of fis $h$ with spots and are few the analyses with groups of stripes. Chromosomally the anostomids with spots represents one of the groups offish beststudied with species showed sex chromosomes (ZZ/ZW) and supernumerary chromosomes, although the majority does not present sex chromosomes. Thus, this study aimed to assist in cytogenetic knowledge of this group of fish through of chromosomal analysis of species Leporellus vittatus and Leporinus striatus. Both species analyzed showed diploid modal value of $2 n=54$ chromosomes distributed in metacentric and submetacentric with fundamental number equal to 108. The constitutive heterochromatin was present in small blocks interstitial or proximal to telomeres of most of chromosomes of L. striatus, with a block heterochromatic well marked in the region pericentromeric a pair of submetacentric corroborating with the nucleolus organizer region (Ag-NOR), type simple. In L. vittatus the constitutive heterochromatin was present in blocks heterochromatic well marked in centromers of some chromosomes while others showed conspicuous markings in the region pericentromeric. The Ag-NOR was evident in a pair chromosome in telomeric region of long arms of one submetacentric chromosome. The results corroborate with those found in other studies, showing that this group has a stable karyotype which is expected in fish that have high vagility.
\end{abstract}

Keywords: Anostomids. Cytogenetics. Chromosomes. Neotropical ichthyofauna. Nucleolus Organizer Region (Ag-NOR).

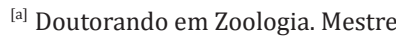
em Ecologia e Conservação da Biodiversidade, Universidade Federal do Paraná (UFPR), Programa de Pós-graduação em Zoologia, Departamento de Zoologia, Setor de Ciências Biológicas, Curitiba, PR - Brasil, e-mail: diones.krinski@ufpr.br

${ }^{[b]}$ Doutor em Genética e Evolução, Universidade Federal do ABC (UFABC), Centro de Ciências Naturais e Humanas, Santo André, SP - Brasil, e-mail: carlosmiyazawa@yahoo.com.br

Recebido: 03/04/2013

Received: 04/03/2013

Aprovado:29/04/2013

Approved: 04/29/2013 


\section{Introdução}

Em comparação com outros grupos de vertebrados, pouco se conhece sobre sistemática, evolução, ecologia, fisiologia, etologia, morfologia e genética de peixes (Böhlke, Weitzman D., Menezes, 1978). Talvez essa falta de conhecimento esteja relacionada ao tipo de habitat que dificulta sua captura, observação e a determinação de seus parâmetros biológicos. Mesmo assim espécies de diferentes famílias de peixes brasileiros são submetidas a estudos citogenéticos e paralelo às diversidades numérica e morfológica. Nota-se que os peixes neotropicais de água doce apresentam considerável variabilidade cromossômica inter e intraespecífica (Oliveira, Toledo, Foresti, Britski \& Toledo-Filho, 1988).

A família Anostomidae compreende peixes popularmente conhecidos como "piaus" na maioria das regiões do Brasil, com 138 espécies descritas e cerca de 25 espécies novas ainda não descritas (Garavello \& Britski, 2003), distribuídas em 12 gêneros. Segundo Garavello e Britski (2003), as espécies desta família são amplamente distribuídas do sul da América Central até o norte da Argentina, exclusivamente na porção cis-andina da América do Sul. É um grupo de peixes com representantes em todas as bacias hidrográficas do Brasil (Géry, 1961; 1977).

Os anostomídeos, em geral, são caracterizados pelo corpo alongado e fusiforme, narina em forma de tubo e um número reduzido de dentes, além de nadadeira anal curta e a dorsal implantada ao nível médio do corpo (com menos de 10 raios ramificados, com exceção de Abramites) (Géry, 1977; Garavello, 1979; Britski, Silimon \& Lopes, 2007; Reis, Kullander \& Ferraris, 2003).

As espécies do gênero Leporellus Lütken, 1874 possuem quatro dentes assimétricos, não cuspidados no pré-maxilar e no dentário, além de lobos da nadadeira caudal recobertos por pequenas escamas. A espécie L. vittatus (Valenciennes, 1850) possui a porção dorsal da cabeça salpicada de pontos escuros e três listras longitudinais ao longo do corpo, que se estendem até a nadadeira caudal, podendo atingir até $30 \mathrm{~cm}$ de comprimento padrão (Britski et al., 2007). Leporinus striatus Kner, 1858, é uma espécie que pode atingir até $25 \mathrm{~cm}$ de comprimento padrão, possui corpo baixo, longo e comprido, é reto na região da inserção da nadadeira anal. Apresenta quatro listras longitudinais ao longo da linha lateral, duas acima, uma ao longo do dorso, e outra abaixo (Britski et al., 2007).
A maioria das espécies da família Anostomidae, já estudadas citogeneticamente, pertencem ao grupo de peixes com pintas, sendo poucas as análises com os grupos de listras. Em relação à composição cromossômica, o grupo dos anostomídeos apresenta-se bem conservado, pois apresentam valor modal de $2 \mathrm{n}=54$ cromossomos, sendo todos metacêntricos e submetacêntricos, apresentando, portanto, número fundamental igual a 108. Dessa forma, poucas diferenças na macroestrutura cromossômicas são encontradas em tais peixes (Galetti-Junior, Cesar \& Venere, 1991; Galetti-Junior, Mestriner, Venere \& Foresti, 1991).

As informações cariotípicas sobre os Anostomidae demonstram que é um dos grupos de peixes mais bem estudados, existindo espécies com cromossomos sexuais do tipo ZZ/ZW e cromossomos supranumerários (Galetti-Junior, Foresti, Bertollo \& MoreiraFilho, 1981a; Galetti \& Foresti, 1986; Mestriner, 1993; Galetti-Junior,Lima\&Venere,1995; Mestriner, Bertollo \& Galetti-Junior, 1995; Koehler et al., 1997; Molina, Schmid \& Galetti-Junior, 1998; Pereira, Oliveira, Foresti \& Maistro, 2002; Venere, Ferreira, Martins \& Galetti-Junior, 2004), embora muitas das espécies cariotipadas não apresentam cromossomos sexuais (Venere, Miyazawa \& Galetti-Junior, 1999; AlmeidaToledo \& Foresti, 2001).

Mesmo considerando a grande quantidade de estudos citogenéticos com peixes anostomídeos, ainda são escassos estudos citogenéticos realizados com estre grupo em rios e riachos da Bacia do Alto Paraguai. Portanto, este trabalho teve como objetivo ampliar o conhecimento cariotípico da família Anostomidae através de análises da macro e microestrutura cromossômica das espécies L. vittatus e L. striatus.

\section{Material e métodos}

As análises cariotípicas foram realizadas em sete exemplares da espécie L. striatus (três machos e quatro fêmeas) (Figura 1a) coletados no rio Sepotuba, região da Cachoeira Salto Maciel $\left(14^{\circ} 41^{\prime} 34,92^{\prime \prime}\right.$; $57^{\circ} 48^{\prime} 13,81^{\prime \prime} \mathrm{W}$ ), município de Tangará da Serra (MT) (Figura 1c), e em oito exemplares da espécie L. vittatus (quatro machos e quatro fêmeas) (Figura $1 \mathrm{~b}$ ) coletados no rio Paraguai $\left(15^{\circ} 04^{\prime} 36^{\prime \prime} \mathrm{S} ; 57^{\circ} 10^{\prime} 54^{\prime \prime} \mathrm{W}\right)$, município de Barra do Bugres/MT (Figura 1d). Ambos os locais de coleta pertencem a bacia do Alto Rio Paraguai. Os espécimes utilizados na obtenção das metáfases cromossômicas foram coletados e enca- 
minhados ainda vivos para o Laboratório de Citogenética Animal da Universidade Federal de Mato Grosso (UFMT), onde permaneceram em aquários até a aplicação das técnicas citogenéticas. Exemplares de
L. striatus e L. vittatus foram fixados em formol $10 \%$ por $24 \mathrm{~h}$, acondicionados em álcool $70 \%$ e depositados na coleção do Laboratório de Citogenética Animal da UFMT, Cuiabá (MT).

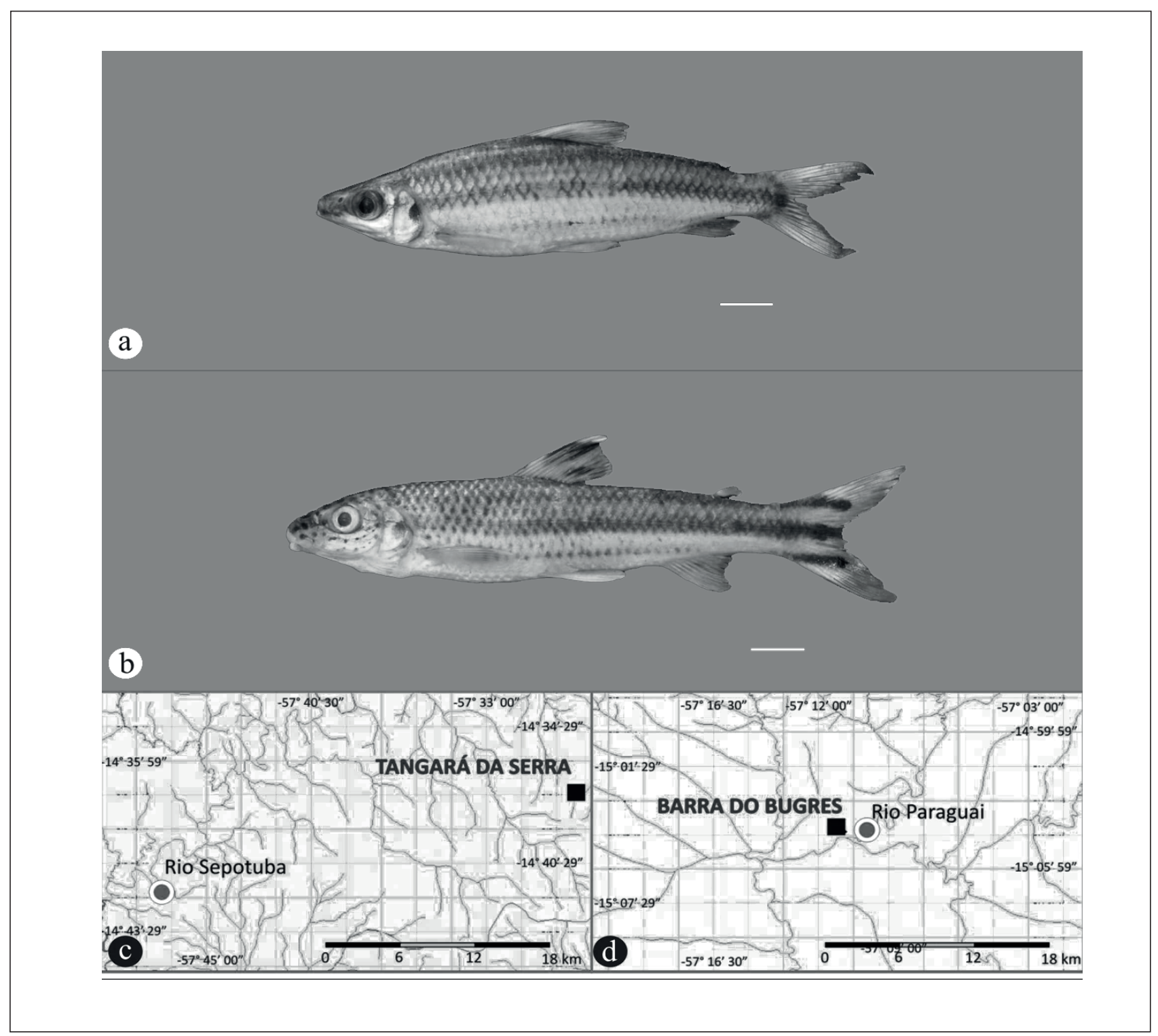

Figura 1 - a) Leporinus striatus; b) Leporellus vitattus. Locais de coleta das espécies; c) Rio Sepotuba - Cachoeira Salto Maciel, Tangará da Serra (MT) (1441'34,92”S; 5748'13,81”W); d) Rio Paraguai, Barra do Bugres (MT) (1504’36”S; 57ำ'54”W)

Fonte: Ministério do Meio Ambiente (Mapa Interativo IGeo - Versão 4.0) - com adaptações. Barras: 1 cm.

Os cromossomos mitóticos foram obtidos a partir de células do rim seguindo a técnica de preparação direta adaptada por Bertollo, Takahashi e Moreira-Filho (1978) para o estudo em peixes. A técnica de banda-C descrita por Sumner (1972) foi empregada para análise da heterocromatina constitutiva.
As regiões organizadoras de nucléolos (Ag-RONs) foram detectadas por coloração de nitrato de prata $\left(\mathrm{AgNO}^{3}\right)$, seguindo método descrito por Howell e Black (1980). A identificação dos cromossomos foi feita de acordo com o critério de relação de braços (RB), sugerida por Levan, Fredga e Sandberg (1964). 


\section{Resultados}

Os indivíduos analisados de L. vittatus, mostraram valor diploide modal de $2 \mathrm{n}=54$ cromossomos, sendo estes metacêntricos e submetacêntricos (NF = 108) (Figura 2a). A heterocromatina constitutiva esteve presente em blocos heterocromáticos bem marcados nos centrômeros de alguns cromossomos enquanto outros apresentaram marcações conspícuas na região pericentromérica (Figura 2b). A região organizadora de nucléolos (RONs) mostrou-se evidente em um par cromossômico na região telomérica do braço longo de um cromossomo submetacêntrico (Figura 2c).

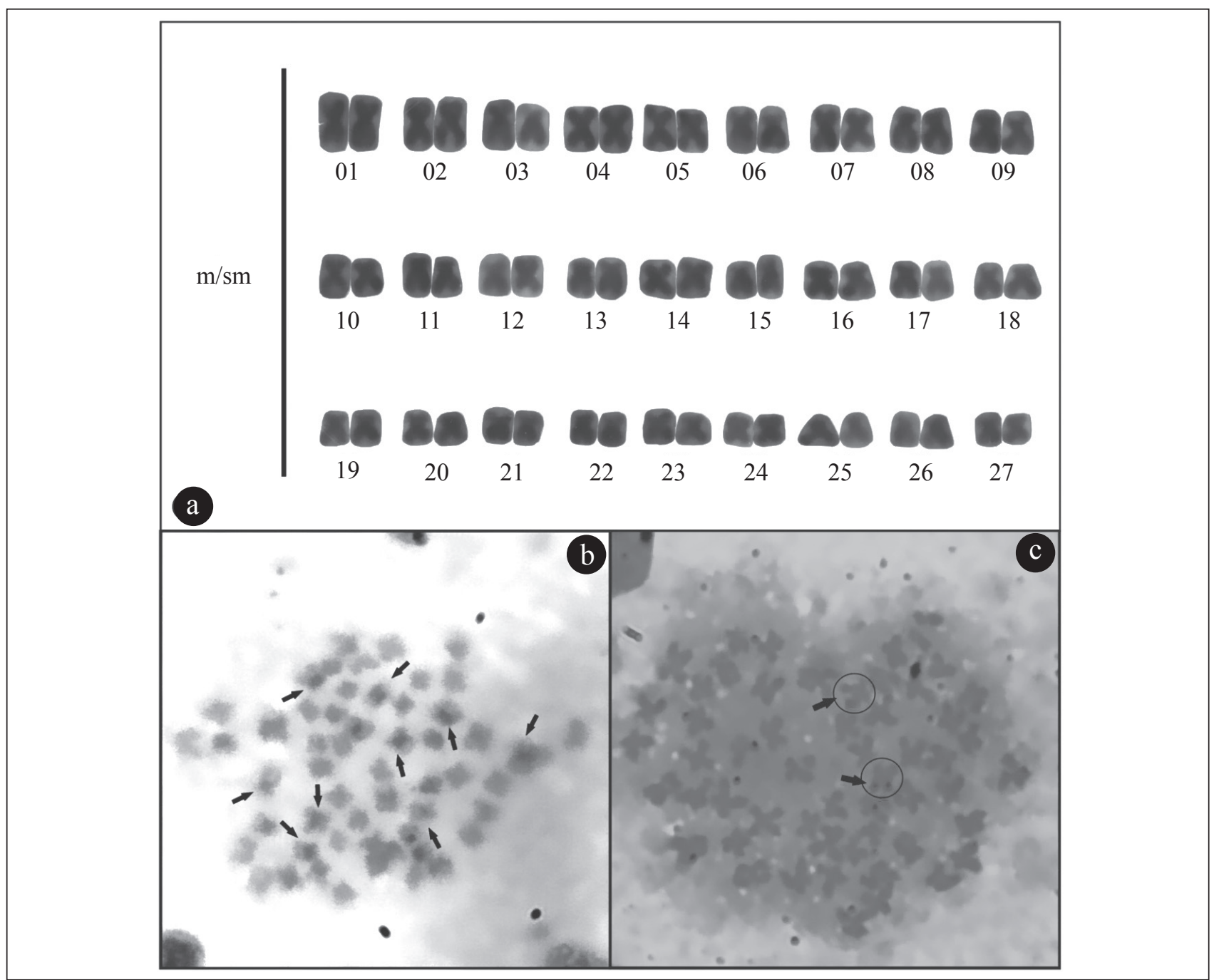

Figura 2 - a) Cariótipo de Leporellus vittatus, evidenciando os pares de cromossomos metacêntricos e submetacêntricos $(2 n=54)$, com número fundamental igual a 108; b) Metáfase mostrando as regiões heterocromáticas (setas), em várias regiões dos cromossomos; c) Regiões organizadoras de nucléolos (círculos)

Fonte: Dados da pesquisa.

Os indivíduos analisados de L. striatus, mostraram valor diploide modal de $2 \mathrm{n}=54$ cromossomos, sendo estes metacêntricos e submetacêntricos e número fundamental igual a 108 (Figura 3a,b). A heterocromatina constitutiva esteve presente em pequenos blocos intersticiais ou proximais aos telômeros da maioria dos cromossomos, havendo um bloco heterocromático bem marcado na região pericentromérica de um par de submetacêntrico e blocos conspícuos também na região pericentromérica de algums cromossomos (Figura 3c). A marcação mais evidenciada pela heterocromática corrobora com a Ag-RON do tipo simples, verificada em apenas um par cromossômico na região pericentromérica (Figura 3d). 


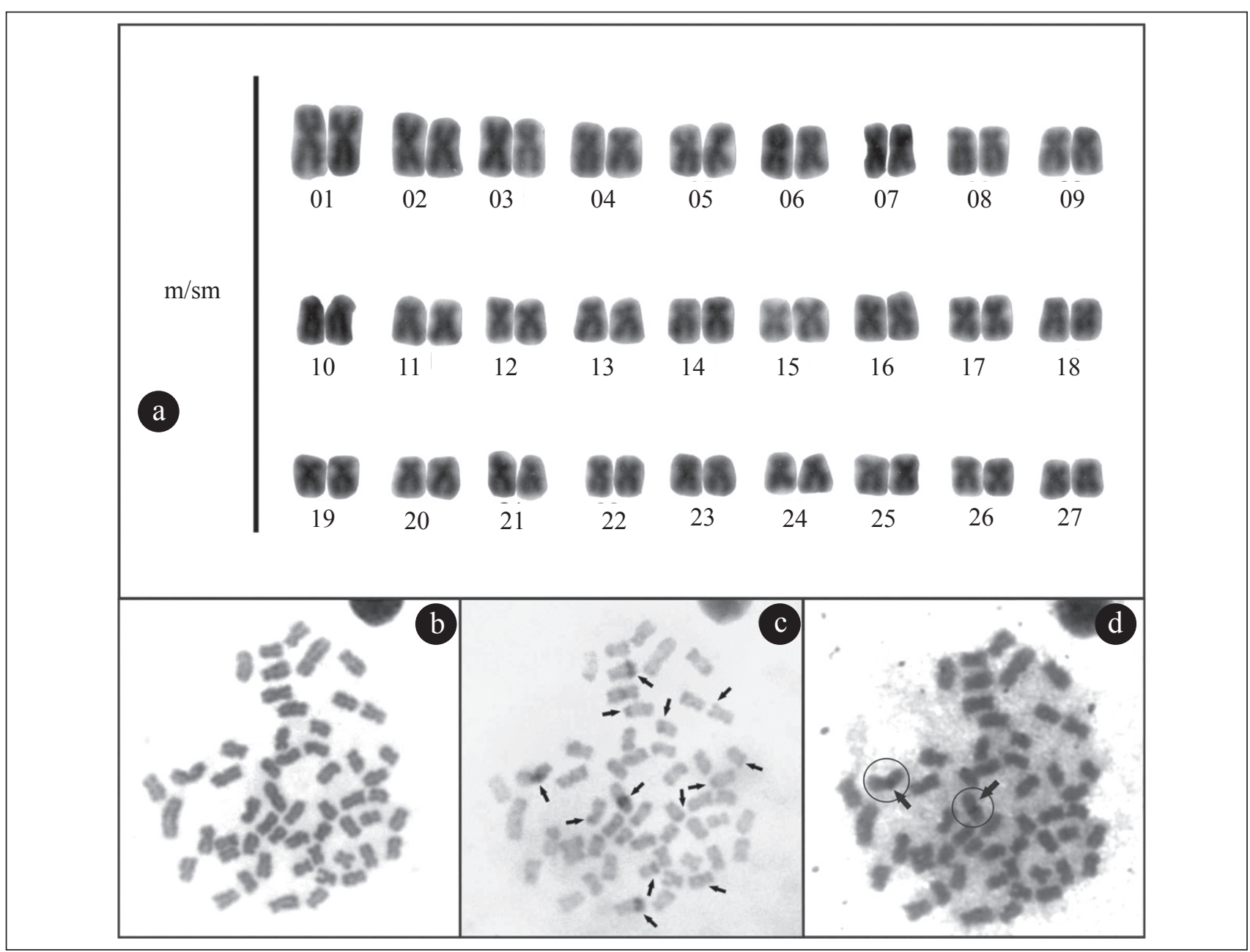

Figura 3 - a) Cariótipo de Leporinus striatus, evidenciando os cromossomos metacêntricos e/ou submetacêntricos (2n = 54) e número fundamental igual a 108; Coloração sequencial; b) Metáfase corada com Giemsa; c) Metáfase mostrando as regiões heterocromáticas (setas), evidenciadas pela técnica de Banda C, em várias regiões dos cromossomos; d) Regiões organizadoras de nucléolos (círculos), evidenciadas pela técnica impregnação por nitrato de prata $\left(\mathrm{AgNO}_{3}\right)$ Fonte: Dados da pesquisa.

\section{Discussão}

Os Characiformes possuem uma grande variação no número cromossômico diploide, sendo distribuídos em dois padrões de diversificação: o primeiro, em que os grupos apresentam grande variabilidade quanto ao número e estrutura cromossômica, como na família Erythrinidae, com relatos de número diploide variando de 39 a 42 cromossomos, e com diversos sistemas de cromossomos sexuais descritos para uma mesma espécie (Hoplias malabaricus); e o segundo, com um padrão contrário, em que os grupos apresentam pequena variação numérica e estrutural do cariótipo como observado nos estudos com Anostomidae, Curimatidae, Prochilodontidae, entre outros (Bertollo,
Born, Dergam, Fenochhio \& Moreira-Filho, 2000; Bertollo, Moreira-Filho \& Galetti-Junior, 1986; Galetti-Junior, Foresti, Bertollo \& Moreira-Filho, 1994).

A partir dos resultados encontrados no presente trabalho, fica evidente que os cariótipos de L. vitattus e L. striatus permanecem estáveis no grupo, tanto na macro quanto na microestrutura cromossômica, a exemplo do que ocorre com outras espécies desta família, que foram estudadas em outras localidades (Galetti-Junior, 1979; Galetti-Junior, Foresti, Bertollo \& Moreira-Filho, 1981ab; Galetti-Junior, Mestriner, Venere \& Foresti, 1991b; Galetti-Junior et al., 1994; Koehler et al., 1997; Martins \& Galetti-Junior, 1998, 1999; Venere, et al., 1999; Martins et al., 2000; Pereira et al., 2002; entre outros). 
Ambas as espécies estudadas apresentaram blocos heterocromáticos na região pericentromérica. Todavia, em algumas espécies do gênero Leporinus, a heterocromatina é praticamente ausente ou, quando presente, apresenta-se em bandas pouco evidentes. Galleti-Junior et al. (1991a) estudando Leporinus piau Fowler, 1941 relatam que a heterocromatina nesta espécie sofreu uma considerável redução, principalmente nas regiões dos centrômeros. Tal padrão também foi encontrado para outras espécies do gênero Leporinus, e parece representar uma unidade derivada do próprio gênero (Garavello, 1979; GalettiJunior et al., 1991b).

Em Leporinus friderici (Bloch, 1794) e Leporinus octofasciatus Steindachner, 1915, a heterocromatina, ocorre em alguns cromossomos na região dos telômeros, com bandas praticamente ausentes na região centromérica (Pereira et al. 2002). 0 mesmo fenômeno é relatado em outro gênero da família Anostomidae, como o exemplo das espécies Schizodon borellii (Boulenger, 1900) e Schizodon isognathu Kner, 1858 (Martins \& Galetti-Junior, 1998).

Segundo Venere et al. (1999) é importante ressaltar que as espécies de Leporinus que apresentam esta redução da heterocromatina têm o mesmo padrão de coloração e pertencem a um mesmo subgrupo. Dessa forma, os resultados de heterocromatina encontrados para L. striatus coincidem com os estudos já realizados neste gênero. No entanto, $L$. vitattus apresentou regiões heterocromáticas próximas ou nos centrômeros, como também observado por Aguilar e Galetti-Junior (2008). Isso indica que esta espécie apresenta-se mais conservada quando comparada com outras espécies da família Anostomidae, que geralmente apresentam poucas regiões heterocromáticas evidentes na região dos centrômeros.

Além da distribuição da heterocromatina nos cromossomos, sua relação com as Ag-NORs é muito diversa entre os peixes e possivelmente indicam diferenças organizacionais envolvendo segmentos cromossômicos (Galetti-Junior, 1998). A maioria das espécies dos Anostomidae apresentam Ag-RONs do tipo simples, com marcações nos cromossomos complementares (Pastori, Fenocchio \& López, 1997; Pereira et al., 2002). Para L. vitattus e L. striatus esta característica não foi diferente, e a semelhança na quantidade de marcações Ag-RONs em espécies distintas conferem uma significância taxônomica como um marcador populacional nesses grupos (Galetti-Junior, Foresti, Bertollo \& Moreira-Filho, 1984).
Dentro dos Characiformes, famílias como Characidae, por exemplo, apresentam uma taxonomia ainda confusa graças à dificuldade em se estabelecer grupos com origem monofilética, diferentemente do que ocorre na família Anostomidae, que tem alta homogeneidade cariotípica, o que sugere uma tendência evolutiva para o grupo (Miyazawa, 1997). Dessa forma, os resultados obtidos corroboram com o encontrado em outros trabalhos, mostrando que esse grupo possui de fato um cariótipo estável, o que é esperado em peixes que apresentam alta vagilidade (Galetti-Junior et al.; 1984, 1991; Aguilar \& Galetti-Junior, 2008).

Os dados são importantes para o acompanhamento da evolução cariotípica nos Anostomidae, porém outros estudos estão em fase de desenvolvimento com técnicas que permitam caracterizar se o grupo possui ou não cromossomos sexuais e de análise da microestrutura cromossômica, como os de Aguilar e Galetti-Junior (2008), que realizaram um mapeamento cromossômico do gene rRNA 5S de diferentes populações de L. vitattus de diversas bacias hidrográficas brasileiras, e a partir dos resultados encontrados sugerem que a espécie atualmente tida como L. vitattus possivelmente compreende um complexo de espécies crípticas.

Então, nota-se que mesmo com os inúmeros estudos realizados com anostomídeos nas diferentes bacias hidrográficas brasileiras, dentre os Characiformes neotropicais, a família Anostomidae merece atenção particular, pois como já ressaltando por Reis et al. (2003), as relações ecológicas e taxonômicas de tal grupo ainda não foram totalmente esclarecidas, além de provavelmente haver um número incerto de espécies descritas. Portanto, as análises citogenéticas podem se tornar uma ferramenta promissora para se utilizar juntamente com dados de sistemática e taxonomia visando auxiliar nos problemas de classificação dentro deste grupo de peixes.

\section{Agradecimentos}

Os autores agradecem a Fundação de Amparo à Pesquisa do Estado de Mato Grosso (Fapemat) pelo suporte financeiro e aos colegas do Laboratório de Citogenética Animal da Universidade Federal de Mato Grosso (UFMT) que acompanharam e auxiliaram durante a realização deste trabalho. 


\section{Referências}

Aguilar, C. T., \& Galetti-Junior, P. M. (2008). Chromosome mapping of 5S rRNA genes differentiates Brazilian populations of Leporellus vittatus (Anostomidae, Characiformes). Genetics and Molecular Biology, 31 (suppl. 1), 188-194.

Almeida-Toledo, L. F., \& Foresti, F. (2001). Morphologically differentiated sex chromosomes in neotropical freshwater fish. Genetica, 111 (1-3), 91-100.

Bertollo, L. A. C., Born, G. G., Dergam, J. A., Fenocchio, A. S., \& Moreira-Filho, O. (2000). A biodiversity approach in the neotropical Erythrinidae fish, Hoplias malabaricus. Karyotypic survey, geographic distribution of cytotypes and cytotaxonomic considerations. Chromosome Research, 8 (7), 603-613.

Bertollo, L. A. C., Moreira-Filho, O., \& Galetti-Junior, P. M. (1986). Cytogenetics and taxonomy: considerations based on chromosome studies of freshwater fish. Journal of Fish Biology, 28 (2), 153-159.

Bertollo, L. A. C., Takahashi, C. S., \& Moreira-Filho, O. (1978). Citotaxonomic considerations on Hoplias lacerdae (Pisces, Erytrinidae). Brazilian Journal of Genetics, 1 (2), 103-120.

Böhlke, J. E., Weitzman, S. H., \& Menezes, N. A. (1978). Estado atual da sistemática dos peixes de água doce da América do Sul. Acta Amozonica, 8 (4), 657-677.

Britski, H.A., Silimon, K, S. \& Lopes, B.S. (2007). Peixes do Pantanal: manual de identificação. 2ed. Brasília: Embrapa.

Galetti-Junior, P. M., Lima, N. R. W., \& Venere, P. C. (1995a). A monophyletic ZW sex chromosome system in Leporinus (Anostomidae, Characiformes). Cytologia, 60 (4), 375-382.

Galetti-Junior, P. M., Mestriner, C. A., Monaco, P. J., \& Rasch, E. M. (1995b). Post-zygotic modifications and intraand inter-individual nucleolar organizing region variations in fish: report of a case involving Leporinus friderici. Chromosome Research, 3, 285-290.

Galetti-Junior, P.M., \& F. Foresti. (1986). Evolution of the ZZ/Zwsystem in Leporinus (Pisces, Anostomidae). Cytogenetic and Cell Genetics, 43 (1-2), 43-46.

Galetti-Junior, P. M., Foresti, F., Bertollo, F. A. C., \& MoreiraFilho, O. (1984). Characterization of eight species of Anostomidae (Cypriniformes) fish on the basis of nucleolar organizer region. Caryologia, 37, 401-406.
Galetti-Junior, P. M., Cesar, A. C. G., \& Venere, P. C. (1991a). Heterochromatin and NORs variability in Leporinus fish (Anostomidae, Characiformes). Caryologia, 44 (3-4), 287-292.

Galetti-Junior, P. M., Mestriner, C. A., Venere, P. C., \& Foresti, F. (1991b). Heterochromatin and karyotype reorganization in fish of the family Anostomidae (Characiformes). Cytogenetic and Cell Genetics, 56 (2), 116-121.

Galetti-Junior, P. M., Foresti, F., Bertollo, L. A. C., \& MoreiraFilho, O. (1981a). Heteromorphic sex chromosomes in three species of the genus Leporinus (Pis-ces, Anostomidae). Cytogenetic and Cell Genetics, 29 (3), 138-142.

Galetti-Junior, P. M.; Foresti, F.; Bertollo , L. A. C., \& MoreiraFilho, O. (1981b). Karyotypic similarity in three genera (Leporinus, Leporellus, Schizodon) of the family Anostomidae (Pisces, Teleostei). Brazilian Journal of Genetics, 4 (1), 11-15.

Galetti-Junior, P. M., Foresti, F., Bertollo , L. A. C., \& MoreiraFilho, O. (1994). Trends in chromosome evolution of neotropical characiform fish. Caryologia, 47 (3-4), 289-297.

Galetti-Junior, P. M. (1998). Chromosome diversity in neotropical. Italian Journal of Zoology, 65 (suppl. 1), 53-56.

Galetti-Junior, P. M. (1979). Estudos citogenéticos na família Anostomidae do rio Mogi-Guaçu, SP (Pisces, Teleos-tei). Dissertação de mestrado, Universidade Federal de São Carlos - UFSCAR, São Carlos, SP.

Garavello, C. J. (1979), Revisão taxonômica do gênero Leporinus - SPIX, 1829 (Ostariophysi, Anostomidae). Tese de doutorado, Departamento de Zoologia, Instituto de Biologia, Universidade de São Paulo - USP, São Paulo, SP.

Garavello, J. C, \& Britski, H. A. (2003). Family Anostomidae (Headstanders). In: R. E. Reis; S. O. Kullander; C. J. Ferraris Jr. Cheklist of the freshwater fishes of South and Central America. (pp. 71-86). Porto Alegre: EDIPUCRS.

Géry, J. (1961). Contributions à l'étude des poissons Characoides. 13. Structures etévolution des Anostominae. Bulletin Aquatics of Biology, 2 (19). 93-112.

Géry, J. (1977). Characoids of the world. Neptune City: T. F. H. Publications. 
Howell, W. M., \& Black, D. A. (1980). Controlled silverstaining of nucleolus organizer regions whit a protective coloidae developer: a l-step method. Experientia, 36 (8), 1014-1015.

Koehler, M. R., Dehm, D., Guttenbach, M., Nanda., I., Haaf, T., Molina, W. F. et al. (1997). Cytogenetics of the genus Leporinus (Pisces, Anostomidae). 1. Karyotype analysis, heterochromatin distribution and sex chromosomes. Chromosome Research, 5, 12-22.

Levan, A., Fredga, K., \& Sandberg, A. A. (1964). Nomenclature for centromeric position on chromossomes. Hereditas, 52 (2), 201-220.

Martins, C., Venere P. C., Mestriner C. A., Cestari M. M., Ferreira R., \& Galetti-Junior P. M. (2000). Chromosome relationships between Anostomidae and Chilodontidae fish (Characiformes). Cytologia, 65, 153-160.

Martins, C., \& Galetti-Junior, P. M. (1999). Chromo-somal localization of 5S rDNA genes in Leporinus fish (Anostomidae, Characiformes). Chromosome Research, 7, 363-367.

Martins, C., \& Galetti-Junior, P. M. (1998). Karyotype similarity between two sympatric Schizodon fish species (Anostomidae, Characiformes) from the Paraguay River Basin. Genetics and Molecular Biology, 21 (3), $355-360$.

Mestriner, C. A. (1993). Análise das regiões organizadoras de nucléolos e investigação do sistema $X X ; X Y$ descrito para Leporinus lacustris (Pisces, Anostomidae). Dissertação de mestrado, Universidade Federal de São Carlos - UFSCar, São Carlos, SP.

Mestriner, C. A., Bertollo, L. A. C., \& Galetti-Junior, P. M. (1995). Chromosome banding and synaptonemal complexes in Leporinus lacustris (Pisces, Anostomidae): analysis of a sex system. Chromosome Research, 3, 440 - 443.

Miyazawa, C. S. (1997). Citogenética de caracídeos da bacia do rio Paraguai: Análises citotaxonômicas-evolutivas e considerações biogeográficas. Tese de doutorado, Universidade Federal de São Carlos - UFSCar, São Carlos, SP.
Molina, W. F., Schmid, M., \& Galetti-Junior, P. M. (1998). Heterochromatin and sex chromosomes in the Neotropical fish genus Leporinus (Characiformes, Anostomidae). Cytobios, 94 (377), 141-149.

Oliveira, C., Toledo, L. F. A., Foresti, F., Britski, H. A., \& Toledo-Filho, S. A. (1988). Chromosome formulae of Neotropical freshwater fishes. Revista Brasileira de Genética, 11 (3), 557-624.

Pastori, M. C., Fenocchio, A. S., \& López, P. A. (1997). First description of microchromosome in the Anostomidae fish Schizodon nasutus from Argentina. Genetics and Molecular Biology, 20 (3), 425-427.

Pereira, M. A., Oliveira, C., Foresti, F., \& Maistro, E. D. (2002). Cytogenetic and nuclear DNA content analysis in Anostomidae fishes from the Sapucaí river, Minas Gerais State, Brazil. Cytologia, 67 (3), 289-296.

Reis, R. E., Kullander, S. O., \& Ferraris-Junior, C. J. (2003). Check list of the freshwater fishes of South and Central America. Porto Alegre, EDIPUCRS.

Sumner, A. T. (1972). A simple techinique for demonstrating centromeric heterocromatin. Experimental Cell Research, 75 (1), 304-306.

Venere, P. C., Miyazawa, C. S., \& Galetti-Junior, P. M. (1999). New cases of supernumerary chromosomes in characiform fishes. Genetics and Molecular Biology, 22 (3), 345-349.

Venere, P. C., Ferreira, I. A., Martins, C., \& Galetti-Junior, P. M. (2004). A novel ZZ;ZW sex chromosome system for the genus Leporinus (Pisces, Anostomidae, Characiformes). Genetica, 121 (1), 75-80. 